\title{
Factores que predisponen a Síndrome de Burnout en personal de enfermería en primer nivel de atención
}

\author{
Factors that Predispose to Burnout Syndrome in Nurses at the First Level of Care
}

\author{
Adelaido Hernández-Hernández ${ }^{1}$, Víctor R. Miranda-Lara ${ }^{2}$, Mayra J. Ramírez-González ${ }^{3}$, \\ Samantha Latorre-Cervantes ${ }^{4}$, Jesús C. Ruvalcaba-Ledezma ${ }^{5}$
}

\begin{abstract}
:
In this review article the factors predisposing to Burnout Syndrome are reported as risk factors in nurses of the first level of care. Aim. To describe the risk factors for Burnout Syndrome in the first level of attention. Methodology. A systematic search was carried out using key words, such as: Burnout in nursing staff, primary care, professional exhaustion, stress in nursing personnel, this search was carried out in Crossref and Pubmed. Results. We reviewed 27 publications, 17 from Crossref (63\%), 10 from PubMed (37\%). Conclusion. High emotional fatigue is the main dimension of affected burnout in primary care nursing. Burnout should be prevented in these professionals by promoting protective factors and monitoring their occurrence in those presenting risk factors.
\end{abstract}

Keywords:

primary health care, professional exhaustion, burnout, risk factors; nursing

\begin{abstract}
Resumen:
En este artículo de revisión se da a conocer los factores que predisponen a Síndrome de Burnout como factores de riesgo en personal de enfermería de primer nivel de atención. Objetivo Describir los factores de riesgo para Síndrome de Burnout en primer nivel de atención. Metodología. Se realizó una búsqueda sistemática mediante palabras clave, tales como: Burnout en personal de enfermería, atención primaria, agotamiento profesional, estrés en personal de enfermería, dicha búsqueda se realizó en Crossref y Pubmed. Resultados. Se revisaron 25 publicaciones, 15 provenientes de Crossref (60\%), 10 de PubMed (40\%). Conclusión. El cansancio emocional alto es la principal dimensión del burnout afectada en la enfermería de atención primaria. Debe prevenirse el burnout en estos profesionales potenciando los factores protectores y vigilando su aparición en los que presenten factores de riesgo.
\end{abstract}

Palabras Clave:

atención primaria de salud, agotamiento profesional, Burnout, factores de riesgo; Enfermería

\section{Introducción}

El Síndrome de Burnout (SB) es un desorden psicológico ampliamente estudiado en las últimas décadas que afecta principalmente a profesionales que trabajan en contacto con personas. Se desarrolla tras una exposición crónica al estrés laboral, y la definición más aceptada es la elaborada por Maslach y Jackson, que lo describen como un síndrome tridimensional donde el trabajador presenta Cansancio Emocional (CE), Despersonalización (D) o

\footnotetext{
${ }^{1}$ Maestrante en Salud Publica, Instituto Elise Freinet, Pachuca, Hidalgo, México. ade821217@ hotmail.com

${ }^{2}$ Centro de Investigación del Estado de Hidalgo, México. vikaras@yahoo.com

${ }^{3}$ Estudiante de Maestría en Ciencias de la Enfermería en Universidad de Guanajuato, México. mayrar642@gmail.com

${ }^{4}$ Residente médico UAEH] Universidad Autónoma del Estado de Hidalgo, México. nicol.32@ hotmail.com

${ }^{5}$ Autor de correspondencia. Investigador de Tiempo Completo en [UAEH] Universidad Autónoma del Estado de Hidalgo, México. Docente 
tratamiento cínico y actitud negativa hacia los usuarios, además de una baja Realización Personal (RP) (2). Para su valoración existen diferentes instrumentos, como el Maslach Burnout Inventory (MBI) o el Cuestionario Burnout Granada (Schaufeli 2009)

Los trabajadores de la salud son los más afectados por el burnout, y son los profesionales de enfermería los que presentan mayor prevalencia (Vargas C. 2015). Se han estudiado procedimientos para el tratamiento del síndrome mediante intervenciones educacionales y meditación, aunque los resultados han sido limitados. El burnout afecta a los profesionales que lo padecen, pero también influye sobre la calidad de los cuidados, los resultados de salud, los pacientes y la propia institución sanitaria.

La actividad laboral es por sí misma generadora de estrés, ya que determina la exposición del empleado a ciertos estresores, que según como sean afrontados, determinarán la respuesta individual, sea adaptativa e implique un aprendizaje y adaptación; o des adaptativa la cual traerá un desgaste e insatisfacción (1).

Algunos estudios han descrito ciertas características que influyen en la salud del trabajador, destacando: el tamaño de la empresa, la responsabilidad que se tiene, las disfunciones de rol y la falta de participación en la toma de decisiones (2).

Así pues, al relacionar estrés y trabajo, surgen exigencias como: necesidad de satisfacción, autoestima y realización personal; las cuales cumplen un papel destacado para encontrarle sentido a la actividad que se realice. Es así como el estrés laboral se puede definir como "El desequilibrio percibido entre las demandas profesionales y la capacidad de la persona para llevarlas a cabo" (3).

En un primer momento, la sociedad británica uso el SB en forma popular dentro de la jerga de los deportistas para describir una situación en la que, en contra de las expectativas de la persona, ésta no lograba obtener los resultados esperados por más que se hubiera preparado y esforzado para conseguirlos.

En castellano, Burnout significa "estar quemado", "síndrome del quemado profesional" o "quemazón profesional”. Según Torres (1997), aludiendo al diccionario de la Real Academia Española, dice que "quemarse" hace referencia a impacientar o desazonar a uno, gastarse, quedarse sin recursos o posibilidades en una actividad cualquiera; y sobre "quemazón" remite a la desazón moral por un deseo no logrado"; estar infeliz y descontento con su labor. Incluso genera síntomas propios de depresión como moral baja, incremento de la irritabilidad, evitación de las relaciones interpersonales y profesionales, baja productividad e incapacidad para soportar la presión. (4)

El termino Burnout fue utilizado por primera vez en el contexto de la Medicina en 1974 por Herbert Freudenberger, un psiquiatra que cuando trató de explicar el deterioro de los cuidados y atención profesional a los usuarios de servicios sanitarios, se percató de la presencia de trastornos emocionales en sus compañeros (5). En 1976, Cristina Maslach usó el término de Burnout como síndrome de "Quemarse en el Trabajo" el cual se caracteriza por presentar en el trabajador: agotamiento físico, psicológico y emocional; baja motivación, actitudes negativas hacia sí mismo y los demás; así como un sentimiento de inadecuación hacia su labor profesional. Todo lo anterior, conduce a que los trabajadores formulen críticas y quejas en cuanto a las condiciones en que desarrollan sus actividades laborales, sus relaciones interpersonales y los obstáculos para su carrera organizacional (6).

En 1977, Maslach dio a conocer esta palabra de forma pública en el Congreso Anual de la Asociación Americana de Psicólogos (APA) describiéndolo como un fenómeno de agotamiento de trabajadores que ejercían su actividad laboral con y para personas, en lo que denominó "trabajadores de servicios humanos", esto es, profesionales que mantienen un contacto constante y directo con personas que son beneficiarias del propio trabajo: docentes, servicios sociales, sanitarios, fuerzas de orden público. En ese sentido, utilizó el término para hacer referencia a una situación de agotamiento, cansancio emocional, desgaste o fatiga psíquica en el trabajo que produce una disminución en la realización personal; la cantidad y calidad del trabajo, así como a la pérdida de energía, sobrepasando y agotando la capacidad del trabajador para afrontar una situación laboral, familiar o social de forma adaptativa.

A principios de los 80, el fenómeno Burnout fue estudiado en EEUU, posteriormente en países de habla inglesa como Canadá y Gran Bretaña; actualmente, podemos encontrar estudios por todo el mundo.

EI SB puede estudiarse desde dos perspectivas, la clínica y la psicosocial. Desde esta última Maslach y Jackson lo definen como un síndrome tridimensional de agotamiento emocional, despersonalización y baja realización personal en el trabajo. (7)

Así pues, el SB, puede reconocerse también como una repuesta al estrés laboral crónico, el cual afecta a las personas cuyo trabajo es ayudar y apoyar a otros; generando en el profesional síntomas que van desde el agotamiento físico, mental y emocional, hasta relaciones conflictivas interpersonales.

La definición más aceptada es la acuñada por Maslach y Jackson, que desde una perspectiva psicosocial comenzaron a estudiar e identificar las distintas condiciones del trabajo que conducen al SB: "es un estrés crónico producido por el contacto con los clientes, el cual lleva a extenuación y distanciamiento emocional con los beneficiarios de su trabajo" (7) completada en los años 80 's como "una pérdida gradual de preocupación y de todo sentimiento emocional hacia las personas con las que trabajan y que conlleva a un aislamiento 0 deshumanización”. Los aspectos básicos que conforman el SB son: cansancio emocional, despersonalización y disminución de la realización personal (8)

El cansancio emocional supone síntomas de pérdida de energía, agotamiento físico y psíquico, fatiga, desgaste y sentimientos de estar al límite, el cual ocurre cuando la fuerza emocional se va consumiendo y el profesional ve cómo se vacía su capacidad de entrega a los demás, desde un nivel personal, físico y psicológico.

La despersonalización implica la aparición de unos sentimientos y actitudes negativas e incluso cínicas acerca del sujeto con el que se trabaja, con un distanciamiento emocional, irritabilidad y rechazo de los mismos. Este proceso, lleva al endurecimiento y a la 
deshumanización de los trabajadores frente a los usuarios a los que atienden, llegando a tratarlos en algunas ocasiones como simples objetos, números 0 incluso considerándolos como merecedores de sus propios problemas.

La disminución de la realización personal; con frecuencia los trabajadores evaden el enfrentamiento y surge la broma o alguna otra forma indirecta de comunicación, lo cual resulta en una descalificación y agresión a las personas.

Así pues, a partir de los 80's, surge una diversidad de libros y artículos en donde planteaban modelos explicativos, se proponen ideas de intervención y se presentaban varias formas de corroborar sus evidencias por medio de cuestionarios, entrevistas y estudios de casos clínicos.

Algunos de los estudios realizados en México; como el de la Ciudad de Mexicali, BC en el Hospital de GinecoPediatría con Medicina Familiar No. 31 y el Hospital General No. 30; donde se estudiaron 368 sujetos; de los cuales $345(93.75 \%)$ son mujeres, con una edad media de 41.21 años (DE 6.59); la antigüedad laboral fue de 15.87 años (DE 6.12). La prevalencia de Burnout en los profesionales de enfermería de ambos hospitales fue del $6.79 \%$. Se concluye que la prevalencia obtenida fue menor a la reportada en otros estudios realizados en México (9)

En el Hospital de las Culturas en Chiapas se realizó un estudio referente al SB en el personal de enfermería; las encuestas fueron aplicadas a un total de 82 trabajadoras con dimensiones de SB observándose: alta carga emocional $95 \%$, con alta despersonalización el $47 \%$ y con bajo logro personal el $100 \%$, por lo que se sugirió desarrollar un programa de gestión para disminuir el estrés ya que está demostrado que esta situación se refleja en la deficiente calidad de atención otorgada a los usuarios del servicio (10)

En un estudio realizado en el hospital General de Pachuca se encontró en el servicio de urgencias un caso con alto índice de agotamiento emocional; mientras que el resto del personal estudiado presenta una falta de sentimiento de realización personal; las puntuaciones más altas provenientes de la calificación del Maslach Inventory Burnout estaban en la clínica de displasias; la Central de Equipos y Esterilización (Ceye), Pediatría, Cirugía General y Urgencias de turno matutino, vespertino, nocturno $A$ y $B$.

Los datos sociodemográficos que presentaron fueron que la edad oscila desde los 30 a 59 años. Meneses estableció que, los estresores provenientes del medio laboral si son determinantes y generadores del inicio del $\mathrm{SB}$, por ejemplo, en los profesionales de la salud la carga de trabajo constante, el tener doble empleo y presentar cansancio emocional por las relaciones en el centro de trabajo; concluyendo que incluso el presentar un nivel bajo de desgaste profesional, es un mecanismo de defensa (8).

Estudios realizados en otros países indican que el profesional de enfermería se expone continuamente a situaciones estresantes en los servicios donde está asignado; como es el caso de las Unidades de Cuidados Intensivos, en donde continuamente se deben tomar decisiones de trascendencia en la atención de urgencia del paciente, según un estudio realizado en Cartagena, establece que a nivel psicológico produce: depresión, ansiedad, riesgo de padecer fatiga por compasión y Burnout (11).

Trabajar en un entorno de contacto continuo con el sufrimiento y la muerte; así como con un gran número de acciones directas e indirectas en donde las enfermeras no pueden expresar sus sentimientos, lo que produce consecuencias negativas para la salud. En otro estudio realizado también en Cartagena, se encontró que la insatisfacción laboral en menores de 40 años se asocia con el desgaste profesional y que la prevalencia del desgaste profesional fue de un $26.6 \%$ (11).

En un estudio realizado en Chile, para determinar la presencia de estrés laboral crónico y su probable relación con factores sociales y laborales en enfermeras que trabajan en Servicios de Atención Médica de Urgencia (SAMU); los resultados mostraron que más del $50 \%$ eran adultos jóvenes, solteros(as) y sin hijos; presentando menos de 10 años de experiencia laboral; la mayoría con hasta cuatro turnos en forma continua. (12)

El grupo de profesionales evidenció presentar SB en una intensidad intermedia, que estaría principalmente influenciado por variables laborales como la percepción de recursos insuficientes y la realización de exceso de turnos. Al mismo tiempo, el grupo de mayor edad evidenció más cansancio emocional; y los viudos o separados presentaron menos despersonalización que el resto de los encuestados (13)

Un estudio realizado en Madrid arroja que la prevalencia del dolor de espalda en el sector hospitalario fue de un $85.8 \%$; lo que implicó consultas médicas en su mayoría $43 \%$, medicación $36 \%$, fisioterapia $27.8 \%$ e interferencias en el trabajo diario $24.6 \%$. Los episodios de dolor se presentaron repetidos $62.2 \%$ y progresivos $52 \%$, siendo su localización por zonas la siguiente: bajó de la espalda $55.2 \%$, nuca $43.8 \%$ y alto de la espalda $42 \%$ (14).

Otros estudios realizados se refieren a la incidencia de accidentes laborales por exposición a material punzocortante asociado a SB como el realizado en el Hospital Nacional "Alberto Sabogal Sologuren Callou", Perú donde se obtuvo el siguiente resultado:

De la población $21.6 \%$ reportó por lo menos un accidente; de los cuales $86.1 \%$ fue de tipo punzocortante y $13.8 \%$ de exposición mucocutánea. Los accidentes se produjeron mayoritariamente en el turno mañana $49 \%$, en las primeras cuatro horas de trabajo $69.1 \%$, en el coche o mesa de preparación de medicamentos $81.1 \%$, durante la administración de medicamentos $55.4 \%$ y antes de la actividad $54.4 \%$. En los accidentes punzocortantes: el $41 \%$ involucró al vidrio de ampolla, el $89.9 \%$ ocurrió en los dedos de las manos y en el $12.5 \%$ hubo presencia de sangre contaminada. En los accidentes de exposición mucocutánea: en su mayoría fue salpicadura en ojos $85.7 \%$ y contacto con orina-saliva-secreciones $77.8 \%$. Reportaron el accidente $10 \%$ de los lesionados. Existió relación entre los accidentes laborales y una media de edad de 32.9 años, así como entre los accidentes y el tiempo de servicio del personalde 7.5 años.

Un estudio realizado en el Hospital Central Militar de México se analizaron 288 reportes de exposición a accidentes laborales, los cuales predominaron en el personal de enfermería, seguido por el grupo médico. Los 
accidentes para el personal de limpieza representaron el $19.09 \%$. Los odontólogos y personal de Rayos X hicieron notificaciones en forma eventual (15)

En un estudio sobre Síndrome de desgaste profesional en trabajadores de atención a la salud en el área sanitaria de Ceuta, se establece que no encuentran diferencias sobre la presencia del síndrome entre enfermeras de áreas críticas, siendo incluso la proporción de personas que presentan desgaste profesional discretamente mayor en las áreas no críticas. En dicho estudio es el hospital, respecto a otros centros de atención primaria 0 de urgencias extrahospitalarias donde se presenta mayor desgaste (16)

La interacción conflictiva, la monotonía de la tarea y la falta de cohesión son variables que influyen en la aparición del síndrome de desgaste profesional. En el caso de las enfermeras del Centro de salud No. 2 de la ciudad de Durango, a mayor interacción conflictiva, mayor monotonía de la tarea y mayor falta de cohesión, mayor será el nivel del síndrome de desgaste profesional que presenten; el síndrome de desgaste profesional ocasiona la aparición de consecuencias psicológicas y organizacionales de manera clara, mientras que en el caso de las consecuencias sociofamiliares y físicas no se presentan con la misma claridad (17).

En un estudio realizado en la Habana Cuba se encontró que en cuanto al estrés profesional, las prevalencias más altas se obtienen en los enfermeros, en particular en los del primer nivel de atención. Entre los médicos, como sucede con el Burnout, es mayor en los que trabajan en el nivel primario, y particularmente en las mujeres. La despersonalización, la falta de realización profesional, el tedio y las características de la tarea tuvieron promedio significativamente más elevados de sus efectos específicos en el primer nivel (66.7\%).

Los síntomas de estrés más frecuentes en los profesionales del primer nivel de atención fueron las cefaleas, ansiedad, irritabilidad, depresión y fatiga. Estos conforman el cuadro típico de los síntomas más significativos, los cuales son señalados como síntomas iniciales de deficiencia de la salud por la Organización Mundial de la Salud (OMS). De todo lo anterior resulta que el perfil de mayor riesgo del SB queda caracterizado en las condiciones médicas de atención primaria, en tanto el correspondiente al estrés, en la enfermera de atención primaria.

Las fuentes de estrés propias de los profesionales de la salud fueron diversas; entre las más señaladas se encuentran la necesidad de actualización de la información especializada, relaciones con los superiores, responsabilidad en el cuidado de los pacientes, bajo reconocimiento de la población asistida (particularmente en enfermeros de nivel primario), incertidumbres éticas en el manejo de la información de los pacientes y el cuidado de las demandas emocionales de estos (18).

En el estudio realizado en el 2013 por este departamento de investigacion respecto a la prevalencia de SB en personal de enfermería de los hospitales de la Secretaria de Salud y del Instituto de Seguridad y Servicios Sociales de los Trabajadores del Estado (ISSSTE) en el Estado de Hidalgo, se encontro que: la frecuencia de Burnout encontrada en los hospitales de la Secretaria de Salud (SSA) y del ISSSTE es alta (33.8\%) con respecto a estudios realizados en otros hospitales del mundo. La prevalencia del síndrome de Burnout fue más alta en las enfermeras que en los enfermeros lo cual ha sido reportado por otros autores.

Resulta preocupante el hecho de que el agotamiento emocional es 4 veces superior en las enfermeras con Burnout y la despersonalización; así como la baja realización personal es prácticamente el doble; lo cual hace necesaria una intervención terapéutica 0 de contención en forma inmediata. Los factores que se asociaron (estadísticamente significativos) fueron el sexo femenino, mayores de 30 años; trabajar en un área crítica del hospital, tener más de 20 años de experiencia laboral y el tener más de dos hijos.

Por otra parte, es importante poner mayor atención en la mejora del ambiente laboral (19.1\% lo consideran desfavorable); a la falta de insumos del hospital (58.8\% dice que son insuficientes); el ausentismo de sus compañeras enfermeras (53.4\% establece que le afecta mucho); así como la necesidad de un espacio digno para descansar (71.3\% lo considera necesario) como factores que favorecen el estrés y el Burnout (19).

Los datos epidemiológicos muestran un incremento de (SB) en el personal de enfermería que afecta su entorno familiar, laboral y de salud. La no intervención hace que permanezcan los trastornos psicosomáticos y de conducta, los cuales se manifiestan en ausentismo y accidentes laborales, por lo cual es necesario conocer la prevalencia del SB en el personal de enfermería de los centros de salud de los servicios de salud de Hidalgo con la intención de generar acciones para mejorar sus condiciones laborales.

\section{CONSIDERACIONES ETICAS}

Normatividad nacional

Clasificación del riesgo de la investigación

De acuerdo al reglamento de investigación en seres humanos en el artículo 17 primera fracción donde se establece que:

Investigación sin riesgo: Son estudios que emplean métodos de investigación documental y retrospectivos y aquellos en los que no se realiza ninguna intervención o modificación intencionada en las variables fisiológicas, psicológicas y sociales de los individuos que participantes en el estudio, entre los que se consideran: cuestionarios, entrevistas, revisión de expedientes clínicos y otros, en los que nos e identifique ni se traten aspectos sensitivos de su conducta.

\section{Discusión}

El síndrome de burnout es consecuencia de eventos estresantes que disponen al individuo a padecerlo. Estos eventos son de carácter laboral, fundamentalmente, ya que la interacción que el individuo mantiene con los diversos condicionamientos del trabajo es la clave para la aparición del síndrome.

Los síndromes de estrés conllevan la defensa o adaptación a exigencias externas. El síndrome de desgaste profesional en trabajadores de atención a la 
salud manifiesta factores intrínsecos (contacto continuado con dolor y muerte, con miedos y angustias, masificación de la actividad, angustia por lagunas e insuficiencias en la formación e incertidumbre en la relación sanitario-paciente) y extrínsecos (condiciones del entorno físico del trabajo, organización del trabajo, exceso de competitividad entre los profesionales e insuficiencia de recursos). (Domínguez Fernández 2012) La prevalencia de síntomas de estrés es mayor en los profesionales de la atención primaria, particularmente en el personal de enfermería femenino, en que se alcanza la mayor prevalencia, que es de 66,7\%.

Los síntomas de estrés más frecuentes en los profesionales del primer nivel de atención son las cefaleas, ansiedad, irritabilidad, depresión y fatiga. Estos conforman el cuadro típico de los síntomas más significativos, los cuales son señalados como síntomas iniciales de deficiencia de la salud por la Organización Mundial de la Salud.

Lo que lleva a una baja productividad en las actividades que realiza el personal de enfermeria, el desinteres en realizar actividades con vocacion y la atencion de calidad y calides hacia la poblacion que demanda sus sevicios.

\section{Conclusiones}

Los datos epidemiológicos muestran un incremento de Síndrome de Burnout en el personal de enfermería que afecta su entorno familiar, laboral y de salud. La no intervención hace que permanezcan los trastornos psicosomáticos y de conducta, los cuales se manifiestan en ausentismo y accidentes laborales, por lo cual es necesario determinar la prevalencia del Síndrome de Burnout en el personal de enfermería de los centros de salud de los Servicios de Salud de Hidalgo con la intención de generar acciones para mejorar sus condiciones laborales y la calidad de la atención a sus usuarios.

La presencia del Síndrome de Burnout en el personal de enfermería de los centros de salud derivado de las condiciones del entorno laboral en el que se encuentran y al tipo de población que se atiende hace necesario que se establezca con claridad las cusas de este con la finalidad de diseñar estrategias de trabajo que promuevan la salud del personal y hacia los usuarios que acuden para recibir atención.

\section{Conflicto de intereses}

Los autores declaramos que no existe conflicto de interés para la publicación del presente artículo.

\section{Referencia}

1. Carmona MFJ, Sanz RLJ, Morin MD. Sindrome de Burnout y reactividad al estres en una muestra de profesionales de Enfermeria en una unidad de cuidados criticos. CIMC. 2000.

2. Mingote AJC, Perez GS. Estres en la enfermeria. El cuidado del cuidador. Diaz de Santos. 2003.

3. Marin C, Fernandez V. Burnout en profesionales de enfermeria Santiago de Chile: Ediciones Universidad Catolica de Chile; 2000.
4. Torres GdCJC. Burnout preofesional: un problema nuevo. Reflexiones sobre el concepto y su evaluacion. Revista de psicologia del trabajo de las organizaciones. 1997.

5. Moreno AS. Prevalencia del Sindrome de Burnout en los Médicos Becarios del HGZ MF No 1, Pachuca Hgo. Pachuca:; 2008.

6. Arévalo CP. Sindrome de Burnout un Problema Actual. Portal psicologico. 2009.

7. Maslach C, Jackson SE. Maslach Burnout Inventory. Journal of Occupational Behaviour. 1997;: p. 99-113.

8. Meneses SL. Sindorme del desgaste profesional (Burnout) en personal de enfermeria de la Secretaria de Salud. Revista Cientifica electronica de Psicologia. 2009.

9. Morales PP, Velez R, Searcy BR, Compean SB. Prevalencia del Sindrome de Burnout en el personal de Enfermería de 2 Hospitales Mexicanos Mexiucali Baja Califrornia. Enfermería Clínica. 2007;: p. 256-260.

10. Ocampo TM, Morales MBE, Guillen , Navarro MdL, Amada dL, Chacón V, et al. Frecuencia de Sindrome Burnout en el Personal de Enfermería del Hospital de las Cuylturas. San Cristobal de las Casas Chiapas:; Junio 2011.

11. Cogollo Z, Milanéz E, Batista C, Cantillo A, Jaramillo, Rodelo D, et al. Desgaste Profesional y Factores Asociados en Personal de Enfermeria Cartagena Colombia. Revista de Enfermería Chia , Colombia. Abril 2010;: p. 3-4.

12. Benjamín VP, Rioseco SP, Saldivia BS, Kohn R, Torres S. Estudio chileno de prevalencia de patologia psiquiatrica. Revista Medica de Chile. 2002.

13. Melito RA. Burnout en profesionales de enfermeria que trabajan en centros asistenciales d la $8^{a}$ region de Chile. Ciencia y Enfermeria. $2008 ;:$ p. $2-14$

14. Nogareda J. Incapacidad temporal personal de enfermeria. Hospital General Universitario de Madrid. 1991.

15. Frias SJA, Hernandez DS, Ruiz CMI. Analisis del Control y Seguimiento del personal de salud expuesto a accidentes laborales Revisón de reportes del Cómite de Infecciones en el Hospital Central Militar. Revista Enf Microbiologia. 2012;: p. 15-24.

16. Dominguez Fernandez JM, Herrera Clavero F, Carmen VGMd, Padilla Segura I, Martinez Bagur ML, Javier DF. Síndrome de desgaste profesional en trabajadores de atención a la salud en el área sanitaria de Ceuta. Atencion Primaria. 2012

17. Barraza Macias A, Carrasco Soto R, Arreola Corral MG. El síndrome de desgaste profesional en las enfermeras del Centro de salud No. 2 de la ciudad de Durango. Revista Psicologia Cientifica. 2008.

18. Hernandez JR. ESTRÉS Y BURNOUT EN PROFESIONALES DE LA SALUD DE LOS NIVELES PRIMARIO Y SECUNDARIO DE ATENCION. Revista Cubana de Salud Publica. 2003;: p. 103-110.

19. Miranda L, Ocampo T, Monzalvo H, Caballero H. Prevalencia de Síndrome de Burnout en personal de enfermería de los hospitales de la Secretaria de Salud y del ISSSTE en el Estado de Hidalgo. Forthcoming. 2014

20. Núnez ZL, Ramirez DR. Caracteristicas Epidemiologicas de los accidentes laborales punzocortantes y de exposición Mucocutanea del personal asistencial de Enfermería. Revista Cientifica de Enfermería. 2000;: p. 10-14.

21. Guonche H, Menendez MN, Angeles APCS, Morales PC, Fonseca SG, Gutierrez GF. Riesgo ocupacional por exposición a objetos punzocortantes en Trabajadores de la Salud. Medicrit Revista de Médicina Interna y Medicina Critica La Habana. 2006;: p. 24-28.

22. Gil_monte PR. Sindrome de quemarse en el trabajo (sindrome de burnout ) en profesionales de nefermeria. In Sindrome de quemarse en el trabajo (sindrome de burnout ) en profesionales de enfermer"ia; 2003; Universidad de Valencia: Revista electronica. p. 26-27.

23. Martínez-López C *LSG*. Características del síndrome de Burnout en un grupO DE ENFERMERAS MEXICANAS. Archivos en Medicina Familiar. Enero Abril 2005;: p. 1. 
24. Fernandez CMV. Éstres entenderlo es manejarlo Santiago de Chile : Ediciones Universidad Catoliuca de Chile ; 2002 2a Edicción.

25. Benjamín Vicente P PRSSSBRKST. Estudio chileno de prevalencia de patologia psiquiatrica. Revista Medica de Chile. 2002. 